\title{
Surface Wave Generation and Propagation on Metallic Subwavelength Structures Measured by Far-Field Interferometry
}

\author{
G. Gay, O. Alloschery, B. Viaris de Lesegno, ${ }^{*}$ and J. Weiner \\ IRSAMC/LCAR, Université Paul Sabatier, 118 route de Narbonne, 31062 Toulouse, France
}

H. J. Lezec

Thomas J. Watson Laboratories of Applied Physics, California Institute of Technology, Pasadena, California 91125 USA and Centre National de la Recherche Scientifique, 3 rue Michel-Ange, 75794 Paris Cedex 16, France

(Received 20 February 2006; published 1 June 2006)

\begin{abstract}
Transmission spectra of metallic films or membranes perforated by arrays of subwavelength slits or holes have been widely interpreted as resonance absorption by surface plasmon polaritons. Alternative interpretations involving evanescent waves diffracted on the surface have also been proposed. These two approaches lead to divergent predictions for some surface wave properties. Using far-field interferometry, we have carried out a series of measurements on elementary one-dimensional subwavelength structures with the aim of testing key properties of the surface waves and comparing them to predictions of these two points of view.
\end{abstract}

DOI: 10.1103/PhysRevLett.96.213901

Early reports of extraordinary optical transmission (EOT) through arrays of subwavelength holes in thin films and membranes [1-3], enhanced well beyond conventional expectation [4] have motivated numerous attempts to explain the underlying physics of these surprising results. Since the early experiments were carried out on metal films, surface plasmon polariton resonances $[5,6]$ were invoked to explain the anomalously high transmission and to suggest new types of photonic devices [6]. Other interpretations based on "dynamical diffraction" in periodic slit and hole arrays $[7,8]$ or various kinds of resonant cavity modes in 1D slits and slit arrays $[9,10]$ have also been proposed. Reassessment of early EOT reports by new numerical studies [11,12] and new measurements [13] have motivated alternative interpretations based on interference and diffraction rather than resonant absorption. The composite diffractive evanescent wave (CDEW) model [13] constructs a surface wave from the distribution of diffracted evanescent modes (the inhomogeneous modes of the "angular spectrum representation" of wave fields [14]) originating at an abrupt surface discontinuity such as a subwavelength-sized hole, slit, or groove when irradiated by a coherent source. The CDEW model exhibits three specific properties. First, the surface wave is considered a composite of modes labeled by the propagation vector component parallel to the surface, and evanescent in the direction normal to the surface. This composite "wave packet" exhibits well-defined, regular nodal positions spaced by a characteristic wavelength, $\lambda_{\text {surf }}$; second, the appearance of the first node at a distance of $\lambda_{\text {surf }} / 2$ from the structured edge implies an effective phase delay of $\pi / 2$ with respect to the $E$ field of the external driving source; and third, an amplitude decreasing inversely with distance from the launch site with an overall effective range of a few microns.

We have fabricated 1D structures (slits and grooves with subwavelength widths) in thin silver films deposited on
PACS numbers: 42.25.Fx, 73.20.Mf, 78.67.- $-\mathrm{n}$

fused silica substrates. The optical response can be studied with the structures facing toward (input-side experiments) or away from (output-side experiments) a distant coherent light source. Results from the input-side experiments, exhibiting transmission interference as a function of slitgroove distance, have been reported elsewhere [15]. We report here measurements of output-side, far-field intensity fringes arising from interference between propagating waves transmitted through the slit and surface waves launched at the slit but, after traveling along the surface, reconverted to outgoing waves at the groove. Studies of fringe frequency, phase, and contrast as a function of slitgroove distance and groove depth provide new and complementary information to the previously reported inputside experiments.

The subwavelength structures are fabricated by focused ion beam (FIB) milling (FEI Nova-600 Dual-Beam system, $\mathrm{Ga}^{+}$ions, $30 \mathrm{keV}$ ) in a $400 \mathrm{~nm}$ thick layer of silver evaporated onto flat fused silica microscope slides. The FIB operating parameters are documented elsewhere [15]. A 2D matrix of structures is milled into the silver layer. Each matrix consists of 63 structures: nine columns, separated by $1.5 \mathrm{~mm}$, and seven rows, separated by $2 \mathrm{~mm}$. The first column contains only slits with no flanking grooves. Variations in transmission through each of the elements in the "slits only" column provide a measure of FIB fabrication uniformity. Light transmission through the slits in this column is used to normalize the transmission in the remaining columns. The remaining structures consist of slits flanked on one side by a groove. The groove-slit distance is systematically increased from the initial to final matrix positions. The square microscope slides themselves are $25 \mathrm{~mm}$ on a side and $1 \mathrm{~mm}$ thick.

Measurements were carried out using a home-built goniometer shown in Fig. 1. Output from a diode laser source $\left(\lambda_{0}=852 \mathrm{~nm}\right)$, temperature stabilized and frequency 


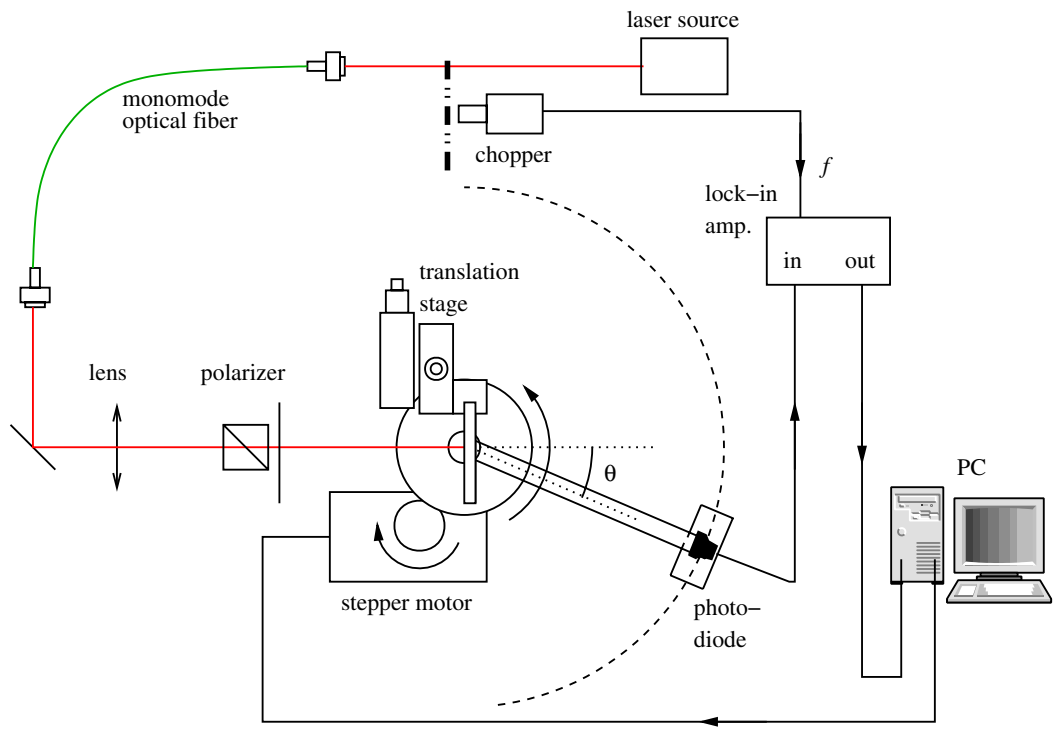

FIG. 1 (color online). Goniometer setup for measuring far-field light intensity and angular distributions. See text for details.

locked is modulated at $850 \mathrm{~Hz}$ by a mechanical chopper, injected into a monomode optical fibre, focused and linearly polarized (TM polarization, $H$ field parallel to the slit) before impinging perpendicularly on the structure matrix mounted in a sample holder. The beam waist diameter and confocal parameter of the illuminating source are $300 \mu \mathrm{m}$ and $33 \mathrm{~cm}$, respectively. Throughout this series of measurements the laser power density was maintained $\sim 1 \mathrm{~W} \mathrm{~cm}^{-2}$. The sample holder itself is fixed to a precision $x-y$ translator, and individual slit-groove structures of the 2D matrix are successively positioned at the laser beam waist. A photodiode detector is mounted at the end of a $200 \mathrm{~mm}$ rigid arm that rotates about an axis passing through the center of the sample holder. A stepper motor drives the arm at calibrated angular increments of $2.05 \mathrm{mrad}$ per step, and the overall angular resolution of the goniometer is $\simeq 4 \mathrm{mrad}$. The photodetector output current passes to a lock-in amplifier referenced to the optical chopper wheel. Data are collected on a personal computer that also controls the goniometer drive.

The structures consist of a single subwavelength slit, $100 \mathrm{~nm}$ wide flanked by one subwavelength groove. Both groove and slit are $20 \mu \mathrm{m}$ long. We have carried out measurements with grooves of two different widths (100 and $415 \mathrm{~nm}$ ) and depths varying from 32 to $256 \mathrm{~nm}$. Here we report results only for the $100 \mathrm{~nm}$ grooves since the wider structures yield similar results.

As illustrated in Fig. 2, the far-field intensity pattern should exhibit interference fringes between $E_{t}$ directly propagating through the slit and $E_{g}$ radiating from the grooves after having been transmitted by the surface waves $E_{\text {surf }}$ launched at the output side of the slit. The frequency and phase of the interference pattern is a function of the optical path difference and any "intrinsic" phase shift (e.g., due to groove shape or surface wave phase lag) of $E_{g}$ with respect to $E_{t}$. Figure 3 shows interference fringes at three representative slit-groove distances $x_{\mathrm{sg}}$ as a func- tion of the goniometer detector angle $\theta$. The fractional surface wave amplitude $\alpha$, normalized to the incoming plane wave amplitude $E_{i}, \alpha=E_{\text {surf }} / E_{i}$, is estimated from the Kowarz model [16] to be about $95 \%$. The remaining $5 \%$ constitutes the amplitude fraction $\delta$ of the light directly transmitted through the slit $E_{t}$. A further fraction $\beta$ is reconverted to propagating light $E_{g}$ at the groove site $x_{\mathrm{sg}}$ and interferes with $E_{t}$. The intensity, $I_{g}$, of the superposed wave fronts can be expressed as

$$
I_{g}(\theta)=\left|E_{t}+E_{g}\right|^{2}=\left|\delta E_{i}+\beta \alpha E_{i} \exp (i \gamma)\right|^{2} .
$$

The normalized intensity $I_{g} / I_{0}$ can then be expressed, with $\eta_{0}=\alpha \beta / \delta$,

$$
\frac{I_{g}}{I_{0}} \propto 1+\eta_{0}^{2}+2 \eta_{0} \cos \gamma, \quad \text { where } \gamma=k_{0} l_{0}+\varphi
$$

with $l_{0}=x_{\mathrm{sg}} \sin \theta \quad$ and $\varphi=k_{\mathrm{surf}} x_{\mathrm{sg}}+\varphi_{\text {int }}$. The relations between $l_{0}, x_{\mathrm{sg}}, \theta$ are shown in Fig. 2 . The frequency and phase of the interference pattern depend on the slit-groove distance through the terms $k_{\text {surf }} x_{\mathrm{sg}}$, and $k_{0} x_{\mathrm{sg}} \sin \theta$. The term $\varphi_{\text {int }}$ represents the intrinsic phase shift due to groove

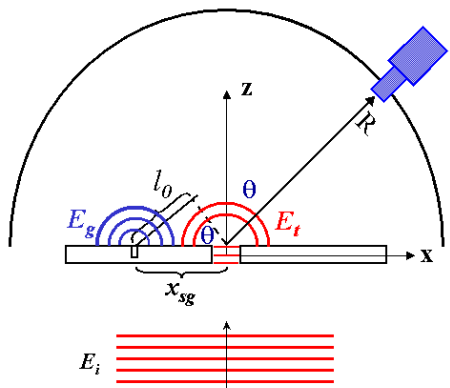

FIG. 2 (color online). Diagram showing interfering wave fronts, optical path difference between $E_{t}$ and $E_{g}$, and far-field detection. The fused silica substrate faces the input side, the metal film the output side; and the slit, groove voids are empty. 


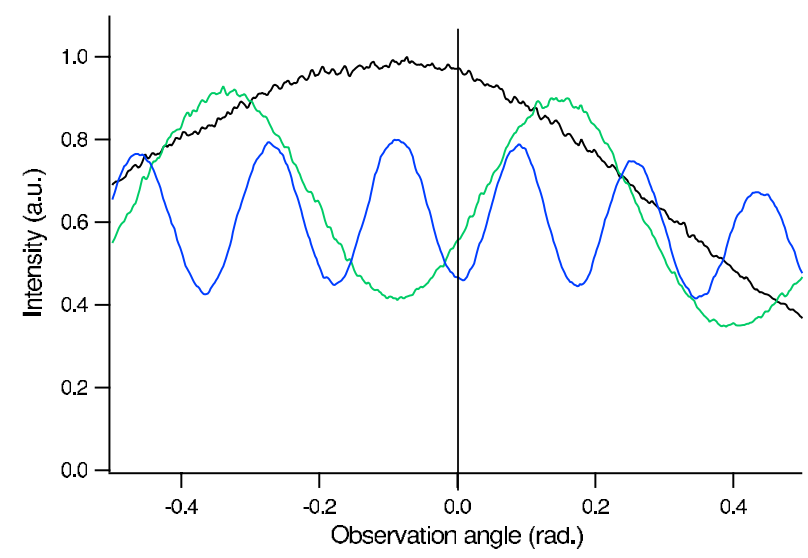

FIG. 3 (color online). Representative output-side interference fringes for structures with $100 \mathrm{~nm}$ wide grooves at three different distances $x_{\mathrm{sg}}$ : black, $0.543 \mu \mathrm{m}$; green, $1.845 \mu \mathrm{m}$; and blue, $4.991 \mu \mathrm{m}$. The phase value at zero observation angle $(\theta=0)$ is used to plot $\cos \varphi$ in Fig. 4.

geometry and surface wave generation at the slit edge. With the goniometer detector oriented perpendicular to the structure plane $(\theta=0)$, Eq. (2) simplifies to

$$
\frac{I_{g}}{I_{0}} \propto 1+\eta_{0}^{2}+2 \eta_{0} \cos \left(k_{\text {surf }} x_{\text {sg }}+\varphi_{\text {int }}\right) .
$$

Figure 4 plots a series of measurements of the far-field intensity as a function of the slit-groove distance $x_{\mathrm{sg}}$ with the detector angle at $\theta=0$. The interference term on the right side of Eq. (3) is the fit to the data from which $\varphi_{\text {int }}$ can be determined by extrapolation of $x_{\mathrm{sg}}$ to zero distance. The magnitude of the surface wave propagation vector $k_{\text {surf }}=$ $2 \pi / \lambda_{\text {surf }}$ is also determined from the fit. We measure $\lambda_{\text {surf }}$ to be $814 \pm 8 \mathrm{~nm}$ in agreement, to within experimental uncertainty, with the input-side experiments reported earlier [15]. The intrinsic phase $\varphi_{\text {int }}$ for this groove geometry

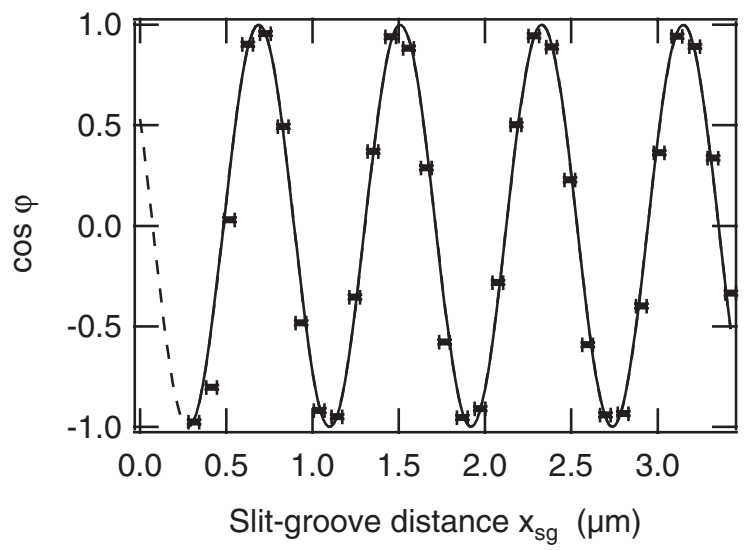

FIG. 4. Plot of the phase $k_{\text {surf }} x_{\text {sg }}+\varphi_{\text {int }}$ as a function of $x_{\mathrm{sg}}$ with the detector oriented perpendicular to the structure plane. Spectral analysis of the frequency spectrum of the fringes yields a determination of the surface wavelength $\lambda_{\text {surf }}=814 \pm 8 \mathrm{~nm}$. Extrapolation of the phase as $x_{\mathrm{sg}}$ approaches zero, yields $\varphi_{\text {int }}=$ $0.32 \pi \pm 0.02 \pi$.
(100 nm width and depth) is determined from the plot to be $\varphi_{\text {int }}=0.32 \pi \pm 0.02 \pi$.

In addition to the frequency and phase of the interference we have studied the "visibility" or contrast of the output-side interference fringes as function of $x_{\mathrm{sg}}$. The interference contrast is defined as

$$
C \equiv \frac{I_{\max }-I_{\min }}{I_{\max }+I_{\min }}
$$

where $I_{\max }, I_{\min }$ are adjacent intensity maxima and minima of the fringes. According to Eq. (2) the contrast can be expressed as

$$
C=\frac{2 \eta_{0}}{1+\eta_{0}^{2}} \quad \text { or } \quad \eta_{0}=\frac{1-\sqrt{1-C^{2}}}{C} \simeq \frac{1}{2} C
$$

Since $\eta_{0} \propto \alpha \beta=E_{g} / E_{i}$, the fractional amplitude radiating at a groove, a plot of $\eta_{0}$ as a function of $x_{\mathrm{sg}}$ measures the dependence of this field amplitude (and therefore the surface wave amplitude) on the slit-groove distance. Figure 5 shows a plot of $\eta_{0}$ as a function of $x_{\mathrm{sg}}$ for narrow-groove structures. The form of the fitted curve through the data points, an inverse distance dependence with an additive constant, is given by Eq. (6) with fitting parameters $\mu, \kappa$ as indicated in the captions of Fig. 5.

$$
\eta_{0}\left(x_{\mathrm{sg}}\right)=\left(\frac{\kappa}{x_{\mathrm{sg}}}+\mu\right) \text {. }
$$

We can now compare these results to predictions of the CDEW model. Two key predictions are (1) that the amplitude of the composite surface wave decreases as the inverse of the distance from the launch site, and (2) that there is an intrinsic phase delay $\varphi_{\text {int }}$ of $\pi / 2$ between $E_{t}$, the directly transmitted wave and $E_{\text {surf }}$ the composite evanescent wave. Figure 5 shows that the contrast (and therefore the ampli-

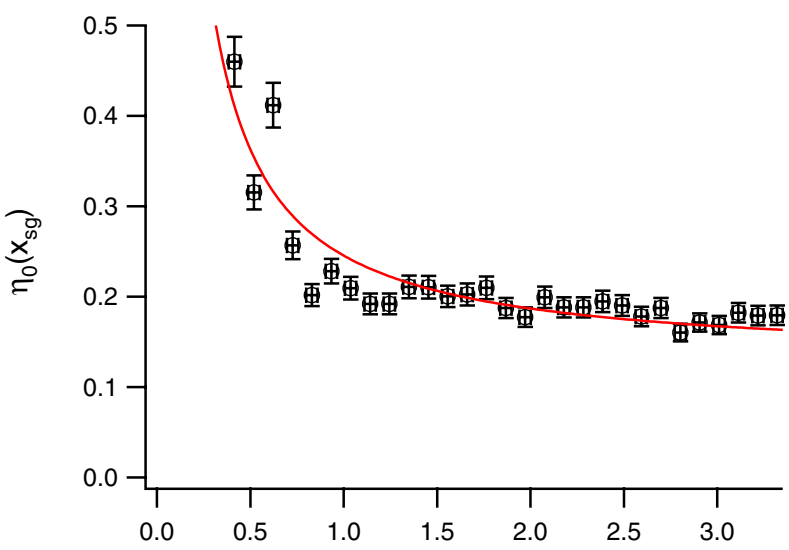

Slit-groove distance $x_{s g}(\mu \mathrm{m})$

FIG. 5 (color online). Plot of $\eta_{0}$ as a function of the slit-groove distance $x_{\text {sg }}$. The quantity $\eta_{0}$ is fit by Eq. (6) with fitting parameters $\mu=0.13 \pm 0.01$ and $\kappa=0.12 \pm 0.01 \mu \mathrm{m}$. 


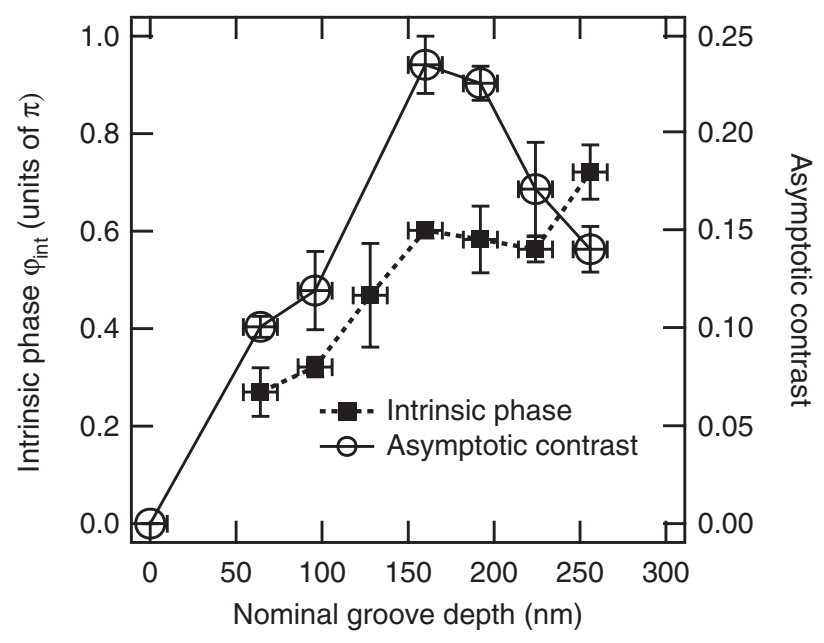

FIG. 6. Intrinsic phase $\varphi_{\text {int }}$ (filled squares, left ordinate) and contrast (open circles, right ordinate) vs groove depth.

tude of $E_{g}$ ) fits well a $1 / x_{\mathrm{sg}}$ behavior for about the first 3 microns, but then stabilizes at a constant contrast.

It appears therefore that there are two components to the surface wave amplitude: a rapidly decreasing component at short range, followed by a constant component at longer range. Figure 4 shows that $\varphi_{\text {int }}$ extrapolates to $0.32 \pi \pm$ 0.02 (not $\pi / 2)$ as $x_{\text {sg }}$ approaches zero. However, it is well known that grooves exhibit "organ-pipe" phase shifts and amplitude resonances when the effective depth is close to an integer number of quarter wavelengths [10]. In order to investigate this contribution to the intrinsic phase we measured the contrast and phase as a function of groove depth. The results are shown in Fig. 6. The contrast indeed shows a maximum near $175 \mathrm{~nm}$ groove depth. Around this resonance the phase lag from the groove must be about modulo $2 \pi$, and therefore any residual intrinsic phase between $E_{t}$ and $E_{g}$ around the groove resonance must be attributed to the phase delay between the surface wave $E_{\text {surf }}$ and the directly transmitted wave $E_{t}$. Figure 6 shows that this residual phase is close to $\pi / 2$, the signature phase lag of the CDEW.

We conclude from these far-field interference phase and contrast results that the surface waves exhibit both CDEWlike and surface plasmon-polariton (SPP)-like properties. The initial, rapid decrease in amplitude and fringe contrast is consistent with a $1 / x$ behavior at small slit-groove distances. The persistance of near-constant contrast at slit-groove distances greater than $\sim 2 \mu \mathrm{m}$ indicates the presence of an SPP-like long-lived mode propagating along the surface. We note, however, that the expected wavelength of the SPP mode on a plane silver surface is $\lambda_{\text {SPP }}=844 \mathrm{~nm}$ [5], but the measured (Fig. 4) $\lambda_{\text {surf }}=$ $814 \pm 8 \mathrm{~nm}$. The reason for the discrepancy is unclear but this "output-side" determination is consistent with the "input-side" results [15]. Furthermore, since the output-side configuration is essentially a Young's doubleslit experiment [17] (with the groove functioning as one of the slits), measurement of the fringes around $\theta=0$ determines the fractional precision of groove-slit distance to $\leq 10^{-3}$, independent of any instrumental FIB calibration. It is also worth emphasizing that, in contrast to the input-side configuration of [15], where both slit and groove are illuminated by the laser source, the angular interference fringes of the present results clearly identify the slit as the source of the surface modes and the groove as the structure reconverting them to propagating modes. Finally, the present study measures intrinsic phase and fringe contrast as a function of groove depth. The results of Fig. 6 indicate that the persistant SPP-like wave conserves the signature phase lag of $\simeq \pi / 2$ when the groove reradiates the surface wave with maximum efficiency around the organ-pipe resonance.

Support from the Ministère délégué à l'Enseignement supérieur et à la Recherche under the programme ACI"Nanosciences-Nanotechnologies," the Région MidiPyrénées [SFC/CR 02/22], and FASTNet [HPRN-CT2002-00304] EU Research Training Network, the Caltech Kavli Nanoscience Institute, and the AFOSR under Plasmon MURI FA9550-04-1-0434 is gratefully acknowledged, as are discussions with P. Lalanne, R. Mathevet, A. Scherer, and H. Atwater.

*Present address: Laboratoire Aimé Cotton, Campus d'Orsay, 91405 Orsay, France.

[1] T. W. Ebbesen, H. J. Lezec, H.F. Ghaemi, T. Thio, and H. J. Wolff, Nature (London) 391, 667 (1998).

[2] T. Thio, K. M. Pellerin, R. A. Linke, H. J. Lezec, and T. W. Ebbesen, Opt. Lett. 26, 1972 (2001).

[3] H. F. Ghaemi, T. Thio, D. E. Grupp, T. W. Ebbesen, and H. J. Lezec, Phys. Rev. B 58, 6779 (1998).

[4] C. J. Bouwkamp, Rep. Prog. Phys. 17, 35 (1954).

[5] H. Raether, Surface Plasmons on Smooth and Rough Surfaces and on Gratings (Springer-Verlag, Berlin, 1988).

[6] W. L. Barnes, A. Dereux, and T.W. Ebbesen, Nature (London) 424, 824 (2003).

[7] M. J. Treacy, Appl. Phys. Lett. 75, 606 (1999).

[8] M. J. Treacy, Phys. Rev. B 66, 195105 (2002).

[9] Q. Cao and P. Lalanne, Phys. Rev. Lett. 88, 057403 (2002).

[10] F. J. García-Vidal, H. J. Lezec, T. W. Ebbesen, and L. Martin-Moreno, Phys. Rev. Lett. 90, 213901 (2003). Note especially Fig. 3 in this reference which shows a transmission resonance at $\lambda=850 \mathrm{~nm}$ and groove depth in the range $175-200 \mathrm{~nm}$, in accord with the results of Fig. 6 reported here.

[11] S.-H. Chang, S. K. Gray, and G. C. Schatz, Opt. Express 13, 3150 (2005).

[12] Y. Xie, A. R. Zakharian, J. V. Moloney, and M. Mansuripur, Opt. Express 13, 4485 (2005).

[13] H. J. Lezec and T. Thio, Opt. Express 12, 3629 (2004).

[14] L. Mandel and E. Wolf, Optical Coherence and Quantum Optics (Cambridge University Press, Cambridge, England, 1995), p. 109.

[15] G. Gay et al., Nature Phys. 2, 262 (2006).

[16] M.W. Kowarz, Appl. Opt. 34, 3055 (1995).

[17] H. F. Schouten et al., Phys. Rev. Lett. 94, 053901 (2005). 